\title{
Chia seeds are richer in polyphenols compared to flax seeds
}

\author{
Oshra Saphier"*, Tali Silberstein ${ }^{2}$, Hila Kamer ${ }^{1}$, Yuval Ben-Abu ${ }^{3}$ and Dorith Tavor ${ }^{1}$ \\ ${ }^{1}$ Department of Chemical Engineering, Sami Shamoon College of Engineering, Beer-Sheva, Israel \\ ${ }^{2}$ Department of Gynecology and Obstetrics, Soroka University Hospital, Ben-Gurion University of the Negev, Beer-Sheva, Israel \\ ${ }^{3}$ Projects and Physics Section, Sapir Academic College, Ashkelon, Israel
}

\begin{abstract}
Background: Polyphenols are the most abundant antioxidants in human diet. The main dietary sources of polyphenols are fruits and plant-derived beverages such as fruit juices, tea, coffee and red wine. Polyphenols are structurally characterized by the presence of one or more phenol units. Flax seeds are rich in phenolic compounds, known as lignans, responsible for its antioxidant activities. Chia seeds exhibit a high antioxidant activity, which is associated with the polyphenolic compounds and the presence of tocopherols.
\end{abstract}

Objectives: (1) To determine total polyphenols in chia seeds compared with flax seeds. (2) To find the optimal conditions for extracting polyphenols from chia and flax seeds using green solvents.

Design: A series of experiments were done in order to determine quantity of total polyphenols: different water: ethanol ratios, different polar index extraction solution, single extraction cycle at delay times ranging from 1-72 hours and one to four extraction cycles. The total amount of seed polyphenol extracts was determined by acolorimetric method by the Folin-Ciocalteu reagent. The amount of polyphenols was determined as equivalents of gallic acid.

Results: Chia seeds are richer in polyphenols than Flax seeds by approximately 42\%. A mixture of water and ethanol of 1:1 generates the highest amount of polyphenols. Polarity index of 7.1 is the most effective in extracting polyphenols. One hour of extraction was found to be the optimal time. It is preferable to perform four cycles of one hour extraction compared to three 72-hour extraction cycles.

Conclusions: Chia seeds, beyond of being a good source of $\omega-3$ linolenic acid, found to be a richer source of polyphenols compared with flax seeds. Mixture of ethanol and water at a 1:1 ratio is most effective for seeds polyphenols extraction. Extractions of one hour in chia and flax seeds are more efficient than extractions of 72 hours.

\section{Introduction}

Changes in human diets over the past 100-150 years, particularly in terms of dietary fat intake and total antioxidants, and its effect on human health, have become a major interest in nutrition research. In recent years, there is a significant increase in the awareness of consumption of food rich in polyphenols as antioxidants. Thus, nutritionists are searching for food sources that are rich in polyphenols.

Polyphenols represent a group of chemical substances that is common in plants and is structurally characterized by the presence of one or more phenol units. Polyphenols are the most abundant antioxidants in human diet. The largest and best studied class of polyphenols is flavonoids which include several thousand compounds. The main dietary sources of polyphenols are fruits and plant-derived beverages such as fruit juices, tea, coffee and red wine. Vegetables, cereals, chocolate, and dry legumes also contribute to the total polyphenol intake [1]. Numerous studies confirm that polyphenols exert a protective action on human health and are key components of a healthy and balanced diet $[1,2]$. Epidemiological studies suggest that long term consumption of diets rich in plant polyphenols offer protection against development of cancers, cardiovascular diseases, diabetes, osteoporosis and neurodegenerative diseases [1].

Flax seeds (Linum usitatissimum) have long been used in human and animal diets as well as in industry where they are used as a source of oil and as the basic component or additive of various paints or polymers. Flax oil is the richest plant source of linoleic and linolenic polyunsaturated fatty acids (PUFA), which are essential for humans
[3]. Flax seeds are also an important source of fiber and protein, which comprise $30 \%$ and $20 \%$ of the seed respectively [4] and is rich in phenolic compounds responsible for its antioxidant activities [5].

Chia seeds (Salvia hispanica L.) were used by the Aztec tribes in the early history of Mesoamerica. This grain crop was important not only for food, but also for medicines and paints. Chia oil is a centuries-old ingredient that has been rediscovered for today's cosmetics and nutritional applications [6]. Chia oil is extremely high in polyunsaturated fatty acids, particularly $\omega-3$ linolenic acid. The main components are linoleic (17-26\%) and linolenic (50-57\%) acids [7]. Chia seeds exhibit a high antioxidant activity which is associated with the polyphenolic compounds and the presence of tocopherols [8].

Epidemiological and scientific evidence have shown a strong relationship among total fat intake and composition and cardiovascular diseases, cancer, diabetes, and depression [9]. However, populations that consume increased amounts of fats rich in $\omega-3$ fatty acids have

Correspondence to: Oshra Saphier, Department of Chemical Engineering, Faculty of Chemical Engineering, Sami Shamoon College of Engineering, BeerSheva Campus: Bialik/Basel Sts., Beer-Sheva 84100, Israel, Tel: 97286475012; E-mail: oshras@sce.ac.il

Key words: polyphenols, folin-ciocalteu method, diet, gallic acid equivalents, omega 3

Received: February 22, 2017; Accepted: March 17, 2017; Published: March 20, 2017 
been shown to have a much lower incidence of coronary heart disease than populations that consume primarily $\omega-6$ fatty acids [10].

Fish and fish oil are the ultimate source of long-chain $\omega-3$ fatty acids. However, all types of fish contain some level of mercury that accumulates in the aquatic food chain as methyl mercury [11]. Therefore the Food and Drug Administration of America (FDA) recommended a list of fishes to be limited in diet, especially for pregnant women, women planning pregnancy, lactating mothers and young children due to high levels of mercury and its effects on the brain and nervous system [12].

Thus, today there is a higher demand for vegetarian sources of omega- 3 fatty acids such as vegetable oil or seeds containing $\alpha$-linolenic fatty acid (ALA) $[13,14]$ One of the richest botanical sources of ALA is the chia seed $[15,16]$. The chia plant is found in a group of annuals in the genera Salvia and Hyptis of the family Labiatae. Species of chia have been cultivated for centuries in Mexico. Protein ranges in concentration from 19.0-26.5\%, oil from 15.9-34.1\%, fiber (ADF) from 22.1-33.4\%, and total dietary fiber (TDF) from 47.1-59.8\% [17].

Today, there is a tendency to replace the flax seeds with chia seeds in daily diet. The reason for that lays on a series of popular reports that chia seeds are more resistant to oxidative damage and rancidity than flax seeds as well as contain higher levels of antioxidants. This popularity is probably the reason why chia seeds are significantly costlier than flax seeds.

The aims of this study were: (1) to find the optimal conditions for extracting polyphenols from chia and flax seeds in order to enable studying the antioxidant properties of chia and flax seeds in extreme conditions, aging, and mechanical processing of seeds. (2) To compare total polyphenols in chia seeds with flax seeds.

\section{Methods}

\section{Extraction of polyphenols}

Samples of $5.0 \mathrm{~g}$ of chia and flax seeds were measured. Seed Samples were grinded for 4 minutes by an electric poppy grinder into a homogenous, fine, and smooth powder. $50 \mathrm{ml}$ of solvents mixture were added to each sample (ethanol and water at various ratios). In order to prevent the oxidation of polyphenols during the extraction process, we added one drop of $\mathrm{HCl}$ (hydrochloric acid) at a concentration of $5 \mathrm{M}$ to each sample. The extraction mixture was shaken in a shaking water bath at room temperature for 72 hours. After 72 hours, Buchner filtering was performed in a vacuum system with a "4 whatman" filtering paper. (Oil free vacuum pump, VP-33, manufactured by $\mathrm{mrc}$ ).

\section{Determination of total polyphenols}

An aliquot of $1.0 \mathrm{ml}$ was taken from the filtrate in order to assay the total polyphenols. The remaining Buchner funnel cake was resuspended in a solution at a volume of $25 \mathrm{ml}$ to perform a second extraction with the same conditions as the first extraction. The third extraction was also performed at a volume of $25 \mathrm{ml}$, and the fourth at $10 \mathrm{ml}$. The total quantity of polyphenols was acquired by the sum total of the four sequential extractions.

The total phenolic contents in the extracts were determined by using the Folin-Ciocalteu method [18]. This method is based on the redox reaction of the reagent, forming a blue color pigment, with typical absorbance at $760 \mathrm{~nm}$. This method of expression is used as a standard for determining the phenol content of various analytes. The total amount of polyphenols was determined as an equivalent of Gallic acid (GAE), mg per $1.0 \mathrm{~g}$ of dry seeds in accordance to the calibration curve for Gallic acid in experimental conditions $(\mathrm{R}=0.99)$. All U.V.Vis measurements were performed using a Cary 100 Bio, UV-Visible spectrophotometer.

\section{Results and Discussion}

\section{Determining optimal solvent mixture for the extraction of polyphenols}

In the first stage of the process, we found an optimal solvent mixture for the extraction of polyphenols from chia and flax seeds. We aimed to do the extraction by using a mixture of ethanol (E) and water (W) since they are environmentally friendly as well as edible. Each extraction was carried out for $1 \mathrm{hr}$, in one cycle, and seed powder mass to solvent volume ratio was kept at 1:10. Figure 1 displays the total polyphenols amount obtained from chia and flax seeds for five different extraction solvents mixtures. In the Figure, DS refers to dry seeds.

There is an absolute preference to extract chia and flax seeds using a mixture of water and ethanol compared to the pure solvents. Mixture of ethanol and water creates a synergistic effect and greatly increases the efficiency of the separation. After four cycles of 72 hour extraction, a bell behavior resulted and the highest level of polyphenols extraction was achieved at an ethanol:water ratio of 1:1. The total polyphenol levels obtained from extraction with a mixture of water:ethanol, 1:1, was about $42 \%$ higher in chia seeds compared with flax seeds. Thus, this ratio was chosen for the following extractions in the study.

\section{The effect of solvent polarity}

It was apparent that the solvents polarity, separated or mixed, have an important role in the separation process. The effect of solution polarity on polyphenols extraction from flax and chia seeds is shown in Figure 2.

Polyphenols extraction is increased depending on the solvent mixture polarity up to a maximum value which was obtained at polarity index of 7.1. Further increase in solvent mixture polarity led to a decline in the amount of polyphenols in the extract. It is likely that high solvent mixture polarity is very important in dissolving alcohols containing - $\mathrm{OH}$ groups because they produce hydrogen bonds with water. However polyphenols also contain a hydrophobic aromatic group. It seems that hydrocarbon residue of ethanol helps dissolve nonpolar parts of polyphenols. It is likely that ethanol has an important role in dissolving the cell membrane and release of cell contents into the solution unlike pure water that hardly allowed the release of polyphenols from powdered seeds. Water is an important component of the solution for its contribution in increasing the index of polarity of the solution which allows for better dissolving of polyphenols.

\section{The effect of time on the extraction}

In order to examine the factor of time upon the extraction, an experiment was conducted where the polyphenol levels were determined in a single extraction cycle at delay times ranging from 1-72 hours. The results are shown in Figure 3.

The results indicate that after one hour in a single extraction cycle, polyphenol saturation is achieved in the mixture. Therefore, one hour of extraction is sufficient.

The effect of consecutive extractions to the total sum of polyphenols

In order to assess the contribution of consecutive extractions to the total sum of polyphenols, two extreme cycle periods were chosen; one 


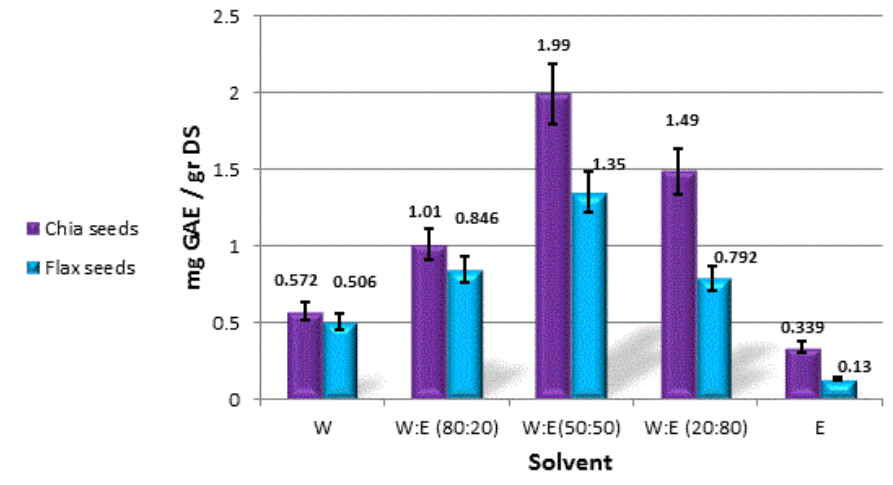

Figure 1. Quantity of total polyphenols (as mg of gallic acid equivalents per $1.0 \mathrm{~g}$ of dry seeds) in chia and flax seeds, obtained from extraction in different water: ethanol ratios

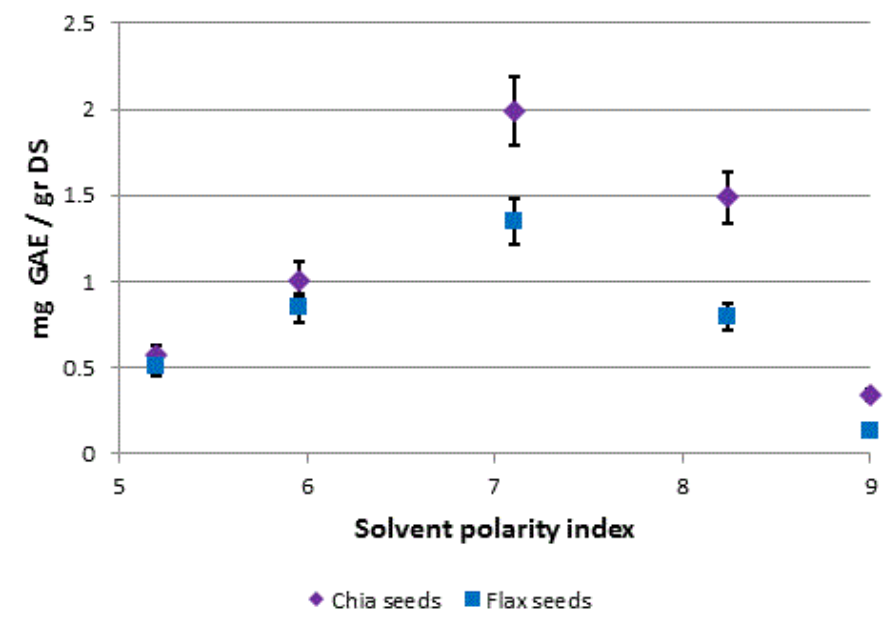

Figure 2. Quantity of total polyphenols (as mg of gallic acid equivalents per $1.0 \mathrm{~g}$ of dry seeds) in chia and flax seeds, depending on the polar index of extraction solution

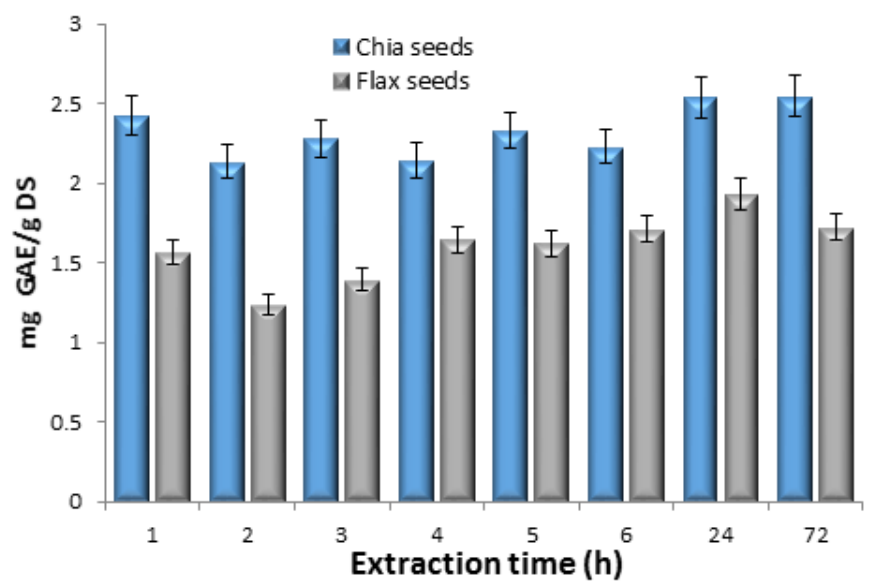

Figure 3. Levels of polyphenols in chia and flax seeds (as mg of gallic acid equivalents per $1.0 \mathrm{~g}$ of dry seeds) as a function of extraction time

hour and 72 hours, and four extraction cycles were performed in each. The results are shown in Figure 4. During both extraction periods an exponential decrease of polyphenol levels was achieved in chia and flax seeds as shown in Figure 5.

One hour extraction cycle resulted in $67 \%$ of the total quantity of polyphenols that was achieved after four extraction cycles in flax and chia seeds. Therefor the amount of total polyphenols can be properly approximated by performing a single one hour extraction cycle and multiplying the result by 1.5 .

The results indicate that it is preferable to perform four cycles of one hour extraction cycles compared to three 72-hour extraction cycles. It is likely that long extraction periods cause oxidation of polyphenols and therefore lower levels are achieved.

One of the criticism on the Folin-Ciocalteu method for assessing the total level of polyphenols arises from the disturbance caused by reducing agents in the system - such as ascorbic acids. Ascorbic acid levels were determined by the absorption of $760 \mathrm{~nm}$ and the creation of an appropriate calibration curve [19]. In chia and flax seeds, a concentration of $0.12 \mathrm{mg}$ of ascorbic acid was found per 1.0 $\mathrm{g}$ of dry seeds. This is extremely low concentration makes it probable that ascorbic acid does not disturb the measurements of polyphenols concentration in chia and flax seeds. Therefore, in this study, as reported in other studies, levels of ascorbic acid are determined without regard to the polyphenols in the examined system.

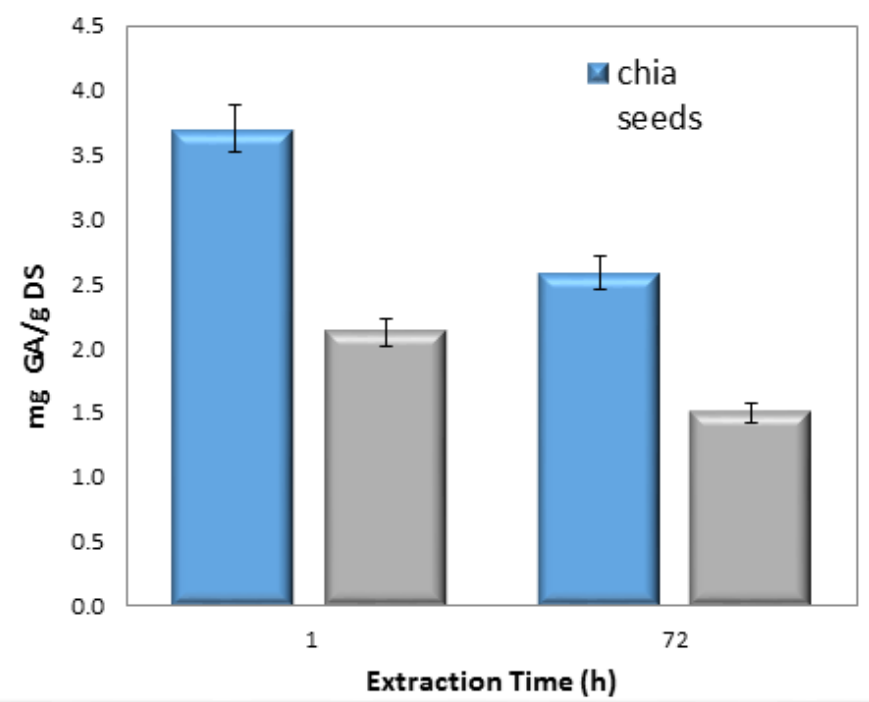

Figure 4. Effect of extraction time on the total polyphenols in chia and flax seeds (as mg of GA per $1.0 \mathrm{~g}$ of dry seeds) in extraction conditions of EtOH-water 1:1.

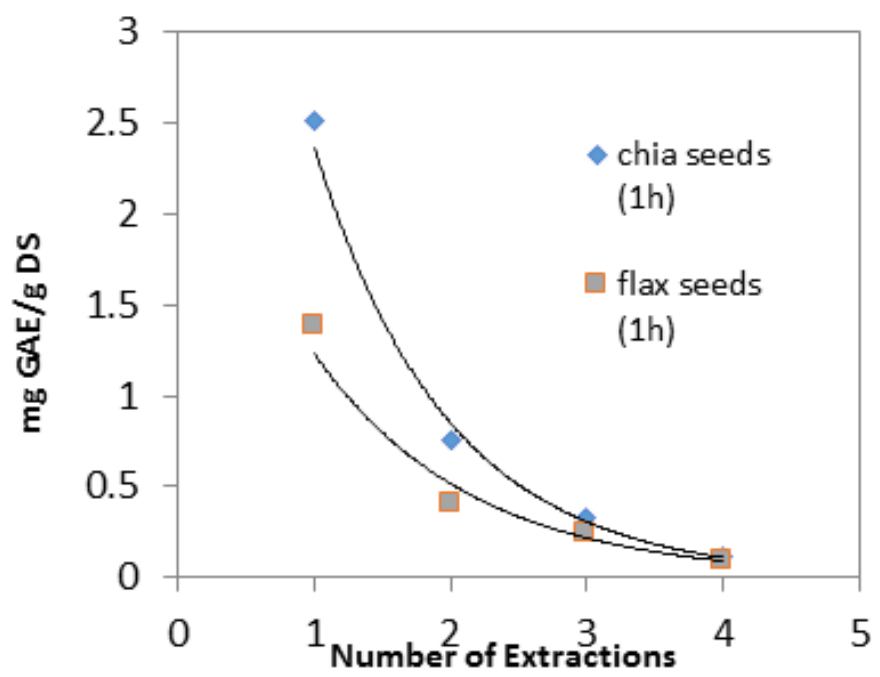

Figure 5a. Levels of polyphenols in chia and flax seeds (as mg of GA per $1.0 \mathrm{~g}$ of dry seeds) as a function of extraction cycles of $1 \mathrm{~h}$ 


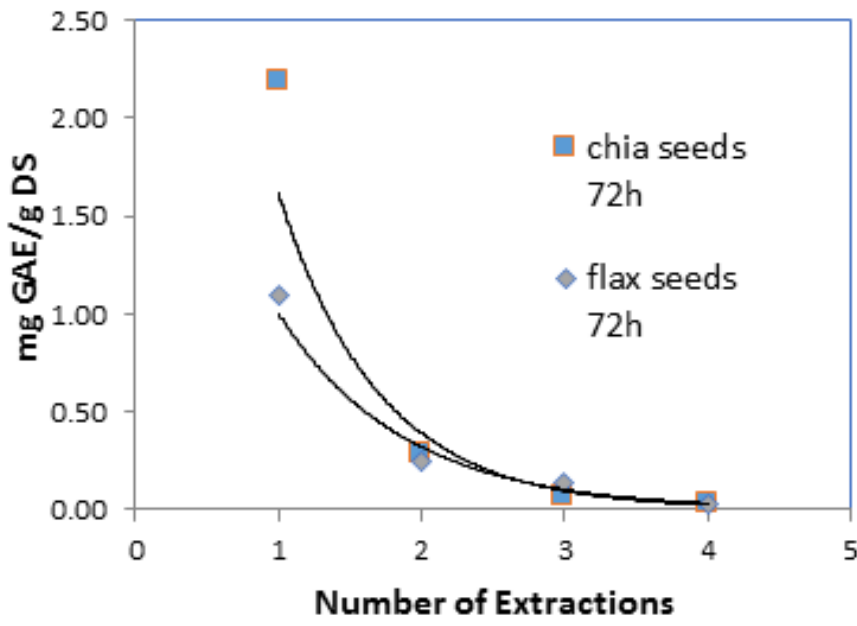

Figure 5b. Levels of polyphenols in chia and flax seeds (as mg of GA per $1.0 \mathrm{~g}$ of dry seeds) as a function of extraction cycles of $72 \mathrm{~h}$

\section{Conclusions}

Chia seeds are richer in polyphenols than flax seeds by approximately $42 \%$. A mixture of ethanol and water at a $1: 1$ ratio is especially effective for the extraction of polyphenols from flax and chia seeds. Unsurprisingly, an exponential relationship is attained between polyphenol levels and the number of extraction cycles. This makes the fourth extraction cycle relatively negligible.

Despite the fact that literature reports the extractions of 72 hours for thyme (Thymus vulgaris L.), sage (Salvia officinalis L.), and marjoram (Origanum majorana L.) extractions [20], we found that extractions of one hour in chia and flax seeds are more efficient than extractions of 72 hours. Thus, if an extraction of polyphenols from seeds is desired, it is advisable to perform four consecutive extractions of one hour per each cycle. In this study we showed that the decline of total polyphenols in seeds powder relative to the extraction cycles is exponential. Therefore, when determination of polyphenols levels is needed, we can predict the total amount of polyphenols in deeds by performing a single onehour extraction cycle and multiply the result by 1.5. This will save time, resources, and reduces environmental concerns.

\section{References}

1. Scalbert A, Johnson IT, Saltmarsh M (2005) Polyphenols: antioxidants and beyond. Am J Clin Nutr 81: 215S-217S. [Crossref]

2. Lima GPP, Vianello F, Corrêa CR, da Silva Campos RA, Borguini MG (2014) Polyphenols in fruits and vegetables and its effect on human health. Food and Nutr Sci 5: 1065-1082.
3. Lukaszewicz M, Szopa J, Krasowska A (2004) Susceptibility of lipids from different flax cultivars to peroxidation and its lowering by added antioxidants. Food chem $88(2)$ 225-231.

4. Omar KA, Shan L, Wang YL, Wang X (2010) Stabilizing flaxseed oil with individual antioxidants and their mixtures. Eur J Lipid Sci Technol 112: 1003-1011.

5. Rubilar M, Gutiérrez C, Verdugo M, Shene C, Sineiro J (2010) Flaxseed as a source of functional ingredients. J Soil Sci Plant Nutr 10(3): 373-377.

6. Ayerza R (1995) Oil content and fatty acid composition of chia (Salvia hispanica L.) from five northwestern locations in Argentina. J AM Oil Chem Soc 72: 1079-1081.

7. Ting IP, Brown JH, Naqvi HH, Kumamoto J, Matsumura M (1990) Chia: a potential oil crop... Proceedings of the first International Conference on New Industrial Crops and Products. The University of Arizona and the Association for The Advancement of Industrial Crops Riverside 197-202.

8. Capitani MI, Spotorno V, Nolasco SM, Tomás MC (2012) Physicochemical and functional characterization of by-products from chia (Salvia hispanica L.) seeds of Argentina. Food Sci Tech 45: 94-102.

9. Okuyama H, Kobayashi T, Watanabe S (1997) Dietary fatty acids-the n-6/n-3 balance and chronic elderly diseases excess linoleic acid and relative n-3 deficiency syndrome seen in Japan. Prog Lipid Res 35: 409-457.

10. Katan MB (2000) Nutritional interventions: the evidence. Proc Nutr Soc 59: 417-418. [Crossref]

11. Barber RT, Whaling PJ, Cohen DM (1984) Mercury in recent and century-old deep-sea fish. Environ Sci Technol 18: 552-555.

12. FDA (1995) FDA Consumer: Cause for Concern? Washington, DC: Food and Drug Administration: 1-7.

13. Mantzioris E, Cleland LG, Gibson RA, Neumann MA, Demasi M, et al. (2000) Biochemical effects of a diet containing foods enriched with n-3 fatty acids. Am J Clin Nutr 72: 42-48. [Crossref]

14. Simopoulos AP (1999) Essential fatty acids in health and chronic disease. Am J Clin Nutr 70: 560S-569S. [Crossref]

15. Webera CW, Gentrya HS, Kohlheppa EA, McCrohanb PR (1991) The Nutritional and Chemical Evaluation of Chia Seeds. Ecology of Food and Nutrition 26: 119-125.

16. Bushway AA, Wilson AM, Houston L, Bushway RJ (1984) Selected properties of the lipid and protein fractions from chia seed. J Food Sci 49: 555-557.

17. Weber CW, Gentry HS, Kohlhepp EA, McCrohan PR (1991). The nutritional and chemical evaluation of chia seeds. Ecology of Food and Nutrition 26: 119-125.

18. Singleton VL, Orthofer R, Lamuela-Raventos RM (1999) Analysis of total phenols and other oxidation substrates and antioxidants by means of Folin-Ciocalteau reagent. Methods Enzymol 299: 152-178.

19. Jagota SK, Dani, HM (1982). A new colorimetric technique for the estimation of vitamin C using Folin phenol reagent. Analytical biochemistry 127: 178-182.

20. Roby MHH, Sarhan MA, Selim KAH, Khalel KI (2013) Evaluation of antioxidant activity, total phenols and phenolic compounds in thyme (Thymus vulgaris L.), sage (Salvia officinalis L.), and marjoram (Origanum majorana L.) extracts. Industrial Crops and Products 43: 827-831. 